$\mathrm{KN}-3$

\section{Understanding and using likelihood in structural biology}

Randy Read ${ }^{1}$, Robert Oeffner ${ }^{1}$, Airlie McCoy ${ }^{1}$

1. Department of Haematology, Cambridge Institute for Medical Research, University of Cambridge, Cambridge, United Kingdom email: rjr27@cam.ac.uk

The principle of maximum likelihood provides an ideal way to understand how experiments and prior knowledge can be used to inform our understanding of the systems we study. Likelihood-based methods now pervade all aspects of macromolecular crystallography and, increasingly, other branches of structural biology.

Compared to previous approaches, such as heuristic methods based on the properties of the Patterson function or least-squares optimisation, likelihood-based methods are found to be more sensitive and to lead to better models. Molecular replacement methods are a prime example where the application of likelihood allows structures to be determined with poorer or less complete models, partly because it provides a clear way to build up more complete solutions from multiple components.

A new understanding of the effects of measurement error [1] and of the statistical properties of crystallographic likelihood targets $[2,3]$ allows us to predict whether or not we will be able to place a particular model, integrating the influence of resolution limit, data quality, model completeness and model quality. This understanding led to the surprising prediction that, given data to sufficiently high resolution, molecular replacement techniques could even be used to place single atoms.

References:

[1] Read, R. J. \& McCoy, A. J. (2016). Acta Cryst. D72, 375-387.

[2] McCoy, A. J., Oeffner, R. D., Wrobel, A. G., Ojala, J. R.,

Tryggvason, K., Lohkamp, B. \& Read, R. J. (2017). Proc. Natl.

Acad. Sci. USA 114, 3637-3741.

[3] Oeffner, R. D., Afonine, P. V., Millán, C., Sammito, M., Uson, I., Read, R. J. \& McCoy, A. J. Acta Cryst. D74, 245-255.

Keywords: likelihood, molecular replacement, phasing
$\mathrm{KN}-4$

\section{How CRISPR-Cas RNA-guided endonucleases cut specific DNA regions for Genome Editing?}

Guillermo Montoya

1. Novo Nordisk Foundation Center for Protein Research Faculty of Health and Medical Sciences, University of Copenhagen, Copenhagen, Denmark

email: guillermo.montoya@cpr.ku.dk

Since ancestral time mankind has tried to modify plants and animals to obtain an offspring that could adapt to our needs. In this way farmers have selected more efficient animals to produce more meat, more resistant plants, faster and resistant horses or cows that produce a larger amount of milk. Our ability to modify organisms has changed dramatically with the introduction of genome editing. Thanks to the discovery of new genome modifying enzymes we can perform a safer and faster manipulation to edit a genome, thus facilitating organism redesign or the treatment of monogenic diseases and some types of cancer. I will address the molecular mechanism that control specificity and cleavage of one of these tools, Cpf1, a single RNA-guided endonuclease of class 2 type V CRISPR-Cas system, which is emerging as a powerful genome editing tool. To provide insight into its DNA targeting mechanism, we have determined the crystal structure of Francisella novicida Cpf1(FnCpf1) in complex with the triple strand R-loop formed after target DNA cleavage. The structure reveals a unique machinery for target DNA unwinding to form a crRNA-DNA hybrid and a displaced DNA strand inside FnCpfl. Our study reveals a singular working model of RNA-guided DNA cleavage by Cpf1, opening up new avenues for engineering this genome modification system.

Keywords: DNA, CRISPR, Genome editing 